Cassandra Calabrese, DO Prabalini Rajendram, MD

Cleveland Clinic
Cleveland Clinic
Gretchen L. Sacha, PharmD

Cleveland Clinic
Leonard Calabrese, DO

Cleveland Clinic

\title{
Practical aspects of targeting IL-6 in COVID-19 disease
}

\section{Posted October 6, 2020}

\section{ABSTRACT}

Interleukin 6 (IL-6) took center stage as a therapeutic target, given its role in the cytokine storm phase of COVID-19. While IL-6 inhibitors have been widely used to treat a variety of immune-mediated disease states, they have not been used often in the intensive care setting, and new data question their efficacy. This brief review provides practical information on their administration and safety.

\section{CURRENT STATUS OF INTERLEUKIN 6-TARGETING}

The natural history of COVID-19 is one of a mildmoderate, self-limiting disease in approximately $80 \%$ of patients, but a more severe disease in the remainder, requiring intensive care in 5\% and carrying a mortality rate of $1 \%$ to $2 \% .{ }^{1}$ In patients with the most severe forms of the disease the course is frequently attended by a syndrome that has been described as "cytokine storm," with some features shared with macrophage activation syndrome. ${ }^{2}$

A variety of experimental therapies are being applied in hospitals around the world targeting this hyperinflammatory state. Early on, interleukin 6 (IL6) took center stage as a therapeutic target given its role in the cytokine storm phase of COVID $-19,{ }^{3}$ and numerous studies have investigated the efficacy of IL-6 inhibitors in this setting. New data have come

Dr. Cassandra Calabrese has disclosed financial interests (consulting/advisory and speaking) with AbbVie, Regeneron, and Sanofi Aventis. Dr. Leonard Calabrese has disclosed financial interests (consulting, teaching, and speaking) with AbbVie, Bristol Myers Squibb, Crescendo Biologics, GlaxoSmithKline, Genentech/ Roche, Horizon Therapeutics, Janssen, Novartis, and Pfizer, Regeneron, Sanofi Aventis, UCB. Dr. Rajendram and Gretchen L. Sacha have nothing to disclose.

The statements and opinions expressed in COVID-19 Curbside Consults are based on experience and the available literature as of the date posted. While we try to regularly update this content, any offered recommendations cannot be substituted for the clinical judgment of clinicians caring for individual patients.

doi:10.3949/ccjm.87a.ccc018 to light questioning the efficacy of this class of targeted therapies, though they are still being utilized in ongoing trials and presumably are being used off-label in extreme circumstances. The currently available agents are listed in Table 1.

While these drugs have been widely used to treat a variety of immune mediated disease states, including cytokine release syndrome secondary to chimeric antigen receptor T-cell (CART) therapy, they have not been frequently used in the intensive care setting. In this review, we provide practical information on their administration and safety.

\section{RATIONALE AND BACKGROUND FOR TARGETING IL-6}

IL-6 is a cytokine with broad-ranging effects on immune function and on a host of nonimmune physiologic functions affecting liver, kidney, central nervous system, muscle, and bone, as well as on glucose and lipid metabolism. ${ }^{4}$ More relevant to COVID19 disease, however, is its central role as a driver of inflammation. C-reactive protein (CRP), a key acute-phase reactant, can be viewed as a downstream secondary messenger for IL- 6 and thus is a reliable biomarker for its activity. ${ }^{4}$

While currently available anti-IL-6 drugs were first approved for autoimmune disorders, tocilizumab was also approved for treatment of cytokine release syndrome accompanying CART therapy of cancer, a syndrome akin to the hyperinflammatory phase of COVID-19 disease. Cytokine dysregulation has been studied in previous viral pneumonias (severe acute respiratory syndrome coronavirus and Middle East respiratory syndrome) and has been associated with increased levels of pro-inflammatory cytokines that lead to T-cell depletion and pulmonary inflammation with extensive lung disease. IL-6 levels were noted to be elevated and correlated with disease severity. ${ }^{5}$ However, serum IL-6 levels are only modestly ele- 


\section{TABLE 1}

Currently available interleukin 6 (IL-6) inhibitors

\begin{tabular}{|c|c|c|c|}
\hline \multirow[b]{2}{*}{ Mechanism of action } & Tocilizumab (Actemra) & Sarilumab (Kevzara) & Siltuximab (Sylvant) \\
\hline & \multicolumn{2}{|c|}{$\begin{array}{l}\text { Tocilizumab and sarilumab: Bind to soluble and membrane-bound IL-6 } \\
\text { receptors and inhibit IL-6-mediated signaling }\end{array}$} & $\begin{array}{l}\text { Binds to IL- } 6 \text { and prevents binding } \\
\text { of IL- } 6 \text { to soluble and membrane- } \\
\text { bound IL- } 6 \text { receptors }\end{array}$ \\
\hline \multirow[t]{5}{*}{$\begin{array}{l}\text { Approved indications } \\
\text { and dosing }\end{array}$} & $\begin{array}{l}\text { Rheumatoid arthritis: } 4 \mathrm{mg} / \mathrm{kg} \\
\text { IV every } 4 \text { weeks (up to } 8 \mathrm{mg} / \mathrm{kg} \\
\text { every } 4 \text { weeks); } 162 \mathrm{mg} \mathrm{SQ} \mathrm{every} \\
\text { other week (up to every week) }\end{array}$ & $\begin{array}{l}\text { Moderately to severely active } \\
\text { rheumatoid arthritis: } 200 \text { mg SQ } \\
\text { every other week }\end{array}$ & $\begin{array}{l}\text { Multicentric Castleman disease: } \\
11 \mathrm{mg} / \mathrm{kg} \text { IV over } 1 \text { hour every } 3 \\
\text { weeks until treatment failure }\end{array}$ \\
\hline & $\begin{array}{l}\text { Giant cell arteritis: } 162 \mathrm{mg} \mathrm{SQ} \text { once } \\
\text { weekly or every other week }\end{array}$ & & \\
\hline & $\begin{array}{l}\text { Polyarticular juvenile idiopathic } \\
\text { arthritis: } 8 \mathrm{mg} / \mathrm{kg} \text { IV every } 4 \text { weeks } \\
(10 \mathrm{mg} / \mathrm{kg} \text { if }<30 \mathrm{~kg}) ; 162 \mathrm{mg} \mathrm{SQ} \\
\text { every other week (every } 3 \text { weeks if } \\
<30 \mathrm{~kg})\end{array}$ & & \\
\hline & $\begin{array}{l}\text { Systemic juvenile idiopathic arthri- } \\
\text { tis: } 8 \mathrm{mg} / \mathrm{kg} \text { IV every other week } \\
(12 \mathrm{mg} / \mathrm{kg} \text { if }<30 \mathrm{~kg}) ; 162 \mathrm{mg} \mathrm{SQ} \\
\text { every week (every other week if < } \\
30 \mathrm{~kg})\end{array}$ & & \\
\hline & $\begin{array}{l}\text { Cytokine release syndrome (due } \\
\text { to chimeric antigen receptor T-cell } \\
\text { therapy): } 8 \mathrm{mg} / \mathrm{kg} \text { IV (10 mg/kg if } \\
<30 \mathrm{~kg} \text { ) }\end{array}$ & & \\
\hline
\end{tabular}

Contraindications Tocilizumab, sarilumab, and siltuximab:

- Safety data are insufficient to recommend use of these agents during pregnancy or breastfeeding

- Use with caution in patients with serious active infection or increased risk of gastrointestinal perforation

Tocilizumab and sarilumab: avoid use in patients with

- $\quad$ ANC $<2,000 / \mathrm{mm}^{3}$

- $\quad$ Platelet count $<100,000 / \mathrm{mm}^{3}$

- $\quad$ ALT/AST > $1.5 \times$ ULN

- Tuberculosis or latent tuberculosis infection

COVID-19 dosing

Current COVID-19

4-8 mg/kg IV for one or two doses (doses given within 24 hours of each other)

$200 \mathrm{mg} \mathrm{SQ}$ once

$11 \mathrm{mg} / \mathrm{kg}$ IV once

Maximum dose: $800 \mathrm{mg} \mathrm{IV}$

clinical trials

NCT04317092, NCT04335071,
NCT04320615, NCT04306705,
NCT04310228, NCT04335305,
NCT04333914, NCT04339712,
NCT04330638, NCT04322773,
NCT04331795, NCT04332094,
NCT04332913, NCT04331808

NCT04341870, NCT04315298,

NCT04327388, NCT04324073,

NCT04329650, NCT04322188,

NCT04321993, NCT04322773 NCT04330638

ALT = alanine aminotransferase; ANC = absolute neutrophil count; AST = aspartate aminotransferase; IV = intravenously; SQ = subcutaneously; ULN = upper limit of normal 
vated in COVID-19 compared with other conditions associated with acute respiratory distress syndrome (ARDS) and often considered under the umbrella of cytokine storm. ${ }^{6}$

\section{RESULTS OF CLINICAL TRIALS}

Over the past several months, numerous nonrandomized studies of IL-6 inhibition in patients with severe COVID-19 infection have been published, and the results have been generally positive. ${ }^{7}$ It should be emphasized, however, that many of these trials suffer from confounders, including inability to account for the progressive improvement in overall management of patients with severe COVID-19, variability in timing of therapy, and use of numerous and nonstandardized concomitant therapies. Similar criticisms have been made of the positive uncontrolled trials of hydroxychloroquine, an agent that has totally failed when put to the test in rigorous randomized controlled trials. ${ }^{8}$ Most importantly, larger, well-designed international randomized controlled trials for tocilizumab (COVACTA, clinicaltrials.gov NCT04320615) and for sarilumab (clinicaltrials.gov NCT04315298) were stopped early for futility. While results of these large clinical trials have not yet been published, their results question the efficacy of IL- 6 for the treatment of COVID-19.

Reasons these studies may have failed include the timing of drug administration and the way patients were stratified into study groups. It is possible that post hoc subgroup analyses could show trends toward efficacy in different patient populations.

Large trials still under way include REMDACTA (NCT04409262), which is evaluating the efficacy and safety of remdesivir plus tocilizumab in patients with severe COVID-19 pneumonia, and the large tocilizumab arm of the Randomised Evaluation of COVID-19 Therapy (RECOVERY) trial (850 patients, and larger than COVACTA), and we anxiously await their results.

At this juncture, we believe there is still reason for the use of anti-IL-6 therapies in clinical trials, but we question its off-label use given the mounting evidence for modest effects and the positive results in the dexamethasone arm of the RECOVERY trial. ${ }^{9}$

\section{PRINCIPLES OF SAFETY}

Targeting IL- 6 carries a series of warnings and safety concerns. Clearly prominent is the increased risk of infection, which attends its chronic use. ${ }^{10}$ IL- 6 inhibitors are associated with rates of serious and opportunistic infections similar to those of other biologic agents, though these data are derived from the chronic rather than the acute application in the current setting. Furthermore, a body of data from preclinical models suggests an important role of IL- 6 in defense against infections in general, and in particular against viral infections. ${ }^{11}$ Most of these models use animals completely deficient in IL-6 signaling, which is different from partial neutralization over a few days.

The infectious complications of IL- 6 inhibition in the acute setting will not be known until a large number of patients are analyzed. Still, an active and uncontrolled secondary bacterial, fungal, or mycobacterial infection would be a strong reason not to embark on a course of IL- 6 inhibition.

IL-6 inhibitors are also associated with an increase in gastrointestinal perforations (1-2 per 100 patientyears compared with tumor necrosis factor inhibitor use), ${ }^{12}$ which may be relevant in patients with acutely decompensating COVID-19; thus, vigilance is warranted. Patients with previous diverticulitis are theoretically at increased risk, but this should not be a contraindication to single use of this therapy in the extreme.

From a laboratory perspective, neutralization of IL-6 can be associated with leukopenia, thrombocytopenia, and elevations of hepatic transaminases, and considerations for discontinuation of the currently available agents are summarized broadly in Table 1 . Chronic administration of anti-IL-6 agents is also associated with perturbations of serum lipids, though this is not a concern in the acute setting. Nevertheless, clinicians using IL-6 inhibitors should be aware of these laboratory associations.

\section{PATIENT SELECTION}

For patients with COVID-19 pneumonia who are rapidly deteriorating with progression to ARDS, there are limited data to direct the management of the hyperinflammatory state. A guidance document by the international task force led by the American Thoracic Society makes no recommendations for or against IL-6 targeting (https://www.thoracic. org/professionals/clinical-resources/disease-relatedresources/covid-19-guidance.pdf). Early on in the pandemic, many centers were actively using IL-6 inhibitors in the treatment of severe COVID-19; however, this has slowed since press releases of COVACTA and the sarilumab study were publicized.

At present, the ideal candidate for IL-6-directed therapy has not yet been defined. Potential candidates are patients with severe pneumonia-classi- 
fied as hypoxemia on room air (peripheral oxygen saturation $<94 \%$ ) and tachypnea (respiratory rate $>30$ breaths per minute), with or without a partial pressure of arterial oxygen to fraction of inspired oxygen ratio $<300 \mathrm{~mm} \mathrm{Hg}$ - and patients with critical pneumonia, defined as requiring mechanical ventilation and involving circulatory shock, with or without multiorgan failure requiring intensive care.

Given the similarities to cytokine release syndrome, a series of biomarkers have been proposed to aid in identifying patients likely to respond and include marked and progressively rising elevations in serum CRP, ferritin, IL-6, and D-dimer, and lymphopenia. ${ }^{13,14}$ Note that IL- 6 in most medical centers is a "send-out" test associated with a delay in obtaining results.

\section{DOSING, ADMINISTRATION, AND RESPONSE}

The majority of patients with COVID-19 treated with anti-IL-6 therapy have received tocilizumab, which is currently labeled for the treatment of CART therapy-induced cytokine release syndrome, giant cell arteritis, polyarticular juvenile idiopathic arthritis, systemic juvenile idiopathic arthritis, and rheumatoid arthritis.

The appropriate dosing regimen of tocilizumab in COVID-19 is currently unknown. A nonrandomized trial by Somers et al, in which 78 COVID-19 patients on mechanical ventilation received tocilizumab, utilized the standard $8-\mathrm{mg} / \mathrm{kg}$ intravenous (IV) dose (maximum $800 \mathrm{mg}$ ). ${ }^{7}$ In cytokine release syndrome due to CART therapy, the recommended IV tocilizumab dose is $8 \mathrm{mg} / \mathrm{kg}$ for patients over $30 \mathrm{~kg}$ (www. gene.com/download/pdf/actemra prescribing.pdf).

A number of trials of tocilizumab in patients with COVID-19 are under way, with doses ranging from 4 to $8 \mathrm{mg} / \mathrm{kg}$, most with a maximum dose of 400 or 800 $\mathrm{mg}$, and most recommending only one dose, but some allowing repeat administration after a specified period of time. Clearly, there is no standardized regimen for this patient population, but dosing regimens of 4 to 8 $\mathrm{mg} / \mathrm{kg} \mathrm{IV} \mathrm{appear} \mathrm{reasonable} \mathrm{at} \mathrm{this} \mathrm{time.}$

\section{EXPECTED EFFECT ON BIOMARKERS}

For now, the most important response to monitor this therapy is clinical, but there is a strong rationale for using appropriate biomarkers to aid in clinical decision-making, both for patient selection and for monitoring response to therapy. As noted above and consistent with known effects of tocilizumab in other cytokine release settings, a number of readily available clinical biomarkers that are abnormal at baseline ${ }^{15}$ can be monitored, including CRP, ferritin, and D-dimer, though the optimal rate and degree of response are yet undefined.

As mentioned above, it is well documented that IL-6 levels will rise in the short term after administration of IL- 6 receptor targeting agents, so it is not appropriate to monitor IL- 6 levels when using tocilizumab. Results of the aforementioned multicenter trials currently under way will provide a rich assessment of clinical and laboratory biomarkers.

\section{CONCLUSIONS}

IL-6 targeting has been widely utilized experimentally and also off-label in selected patients with COVID19 who are sick and deteriorating. Yet it is currently an unproven and unapproved for therapy of COVID19 , and its place in treatment is less than certain. We continue to eagerly await the ability to define the standard of care, which will ultimately only be determined by properly performed trials.

\section{REFERENCES}

1. Wu Z, McGoogan JM. Characteristics of and important lessons from the coronavirus disease 2019 (COVID-19) Outbreak in China: summary of a report of 72,314 cases from the Chinese Center for Disease Control and Prevention. JAMA 2020; 323(13):1239-1242. doi:10.1001/jama.2020.2648

2. Calabrese LH. Cytokine storm and the prospects for immunotherapy with COVID-19. Cleve Clin J Med 2020; 87(7):389-393. doi:10.3949/ ccjm.87a.ccc008

3. Coomes EA, Haghbayan H. Interleukin-6 in COVID-19: a systematic review and meta-analysis. Rev Med Virol 2020; e2141. doi:10.1002/ rmv. 2141

4. Calabrese LH, Rose-John S. IL-6 biology: implications for clinical targeting in rheumatic disease. Nat Rev Rheumatol 2014; 10(12):720727. doi:10.1038/nrrheum.2014.127

5. Sarzi-Puttini P, Giorgi V, Sirotti S, et al. COVID-19, cytokines and immunosuppression: what can we learn from severe acute respiratory syndrome? Clin Exp Rheumatol 2020; 38(2):337-342. pmid:32202240

6. Sinha P, Matthay MA, Calfee CS. Is a "cytokine storm" relevant to COVID-19? JAMA Intern Med 2020; doi:10.1001/ jamainternmed.2020.3313

7. Somers EC, Eschenauer GA, Troost JP, et al. Tocilizumab for treatment of mechanically ventilated patients with COVID-19. Clin Infect Dis 2020; doi:10.1093/cid/ciaa954

8. Shah RR. Chloroquine and hydroxychloroquine for COVID-19: Perspectives on their failure in repurposing. J Clin Pharm Ther 2020; doi:10.1111/jcpt.13267

9. Dexamethasone in hospitalized patients with COVID-19-preliminary report. N Engl J Med 2020 Jul 17. doi:10.1056/NEJMoa2021436

10. Pawar A, Desai RJ, Solomon DH, et al. Risk of serious infections in tocilizumab versus other biologic drugs in patients with rheumatoid arthritis: A multidatabase cohort study. Ann Rheum Dis 2019; 78(4):456-464. doi:10.1136/annrheumdis-2018-214367

11. Velazquez-Salinas L, Verdugo-Rodriguez A, Rodriguez LL, Borca MV. The role of interleukin 6 during viral infections. Front Microbiol 2019 May 10; 10:1057. doi: 10.3389/fmicb.2019.01057

12. Monemi S, Berber E, Sarsour K, et al. Incidence of gastrointestinal perforations in patients with rheumatoid arthritis treated with 
tocilizumab from clinical trial, postmarketing, and real-world data sources. Rheumatol Ther 2016; 3(2):337-352. doi:10.1007/ s40744-016-0037-z

13. McGonagle D, Sharif K, O'Regan A, Bridgewood C. The role of cytokines including interleukin-6 in COVID-19 induced pneumonia and macrophage activation syndrome-like disease. Autoimmun Rev 2020; 19(6):102537. doi:10.1016/j.autrev.2020.102537

14. Cron RQ, Chatham WW. The rheumatologist's role in COVID-19. J Rheumatol 2020; 47(5):639-642. doi:10.3899/jrheum.200334

15. Gao Y, Li T, Han M, et al. Diagnostic utility of clinical laboratory data determinations for patients with the severe COVID-19. J Med Virol 2020; 92(7):791-796. doi: 10.1002/jmv.25770

Correspondence: Cassandra Calabrese, DO, Department of Rheumatic and Immunologic Diseases, A50, Cleveland Clinic, 9500 Euclid Avenue,

Cleveland, OH 44195; CALABRC@ccf.org 